\title{
Similar but not the same: A comparison of the utility of directly rated and feature-based similarity measures for generating spatial models of conceptual data
}

\author{
Matthew J. DRY AND Gert Storms \\ University of Leuven, Leuven, Belgium
}

\begin{abstract}
Spatial models are employed to represent conceptual data in a wide range of fields within psychological research. In order to generate spatial models, it is necessary to first obtain empirical similarity data. A number of methods are available for collecting these data, but little effort has been made to compare their relative utility. In this article, we compare directly rated and five feature-based similarity data types in regard to their ability to be adequately represented by a spatial model (representational goodness of fit), and the ability of the representations to predict three external empirical variables (predictive validity). The results indicate that the representational goodness of fit of the feature-based similarities is noticeably superior to the directly rated similarities, and that the predictions of representations derived from common feature similarity data are substantially more likely than the predictions of all of the alternative representations. It is suggested that these findings are highly relevant to researchers employing spatial models to represent conceptual data, given that direct pairwise ratings have generally been considered the "gold standard" means of obtaining empirical similarities.
\end{abstract}

Spatial models of conceptual data represent stimuli as points in a multidimensional psychological space. These models are commonly generated using multidimensional scaling (MDS; Kruskal, 1964a, 1964b; Shepard, 1962a, 1962b), which requires as input a matrix of empirical data defining the similarity (or dissimilarity) between each of the $n$ stimuli within a set and all of the other $n-1$ stimuli within the set. Given the input data, the multidimensional scaling algorithm searches for the model (within a given dimensionality) that best represents the data such that stimuli that have a high degree of similarity tend to be located close to each other within the space, and dissimilar stimuli located far apart. For example, within a twodimensional spatial representation of the category mammals (such as can be seen in Figure 1), lion and tiger could be expected to be located closer to each other than monkey and hippopotamus.

The use of spatial models as a means of representing conceptual data has been applied in a wide range of research fields within psychology, including (but not restricted to) personality and social psychology (e.g., Ashmore, Griffo, \& Green, 2007; McCallum, McCallum, \& Gurwitch, 1987; Rosenberg \& Jones, 1972), risk perception and public health (e.g., Boverie, Scheufele, \& Raymond, 1994; Goodwin et al., 2004), neuropsychol- ogy (e.g., Elevåg \& Storms, 2003; Prescott, Newton, Mir, Woodruff, \& Parks, 2006), and developmental psychology (e.g., Miller \& Gelman, 1983; Schwanenflugel, Fabricius, \& Noyes, 1996), as well as research dealing specifically with the acquisition and storage of semantic concepts and categories in memory (see, e.g., Shoben \& Ross, 1987, for an overview of the early literature in this field).

Although early research was focused primarily on the use of spatial models for descriptive purposes (e.g., Clark, 1968; Fillenbaum \& Rappaport, 1971; Henley, 1969), numerous subsequent studies have shown that spatial representations also have a high degree of predictive utility. For example, Rosenberg and Jones (1972) used co-occurrence data to generate spatial representations of 99 physical and psychological traits. They then regressed empirical ratings of these traits across 12 properties onto the coordinates of the traits within the space and found increasing predictive multiple correlations as the dimensionality of the representation increased from one to four dimensions. Furthermore, it has been demonstrated that spatial representations can reliably capture the graded typicality structure present in many semantic categories (e.g., Ameel \& Storms, 2006; Rosch \& Mervis, 1975; Verheyen, Ameel, \& Storms, 2007), and similar approaches have also been applied to the prediction of categorization response times

M. J. Dry, matt.dry@psy.kuleuven.be 


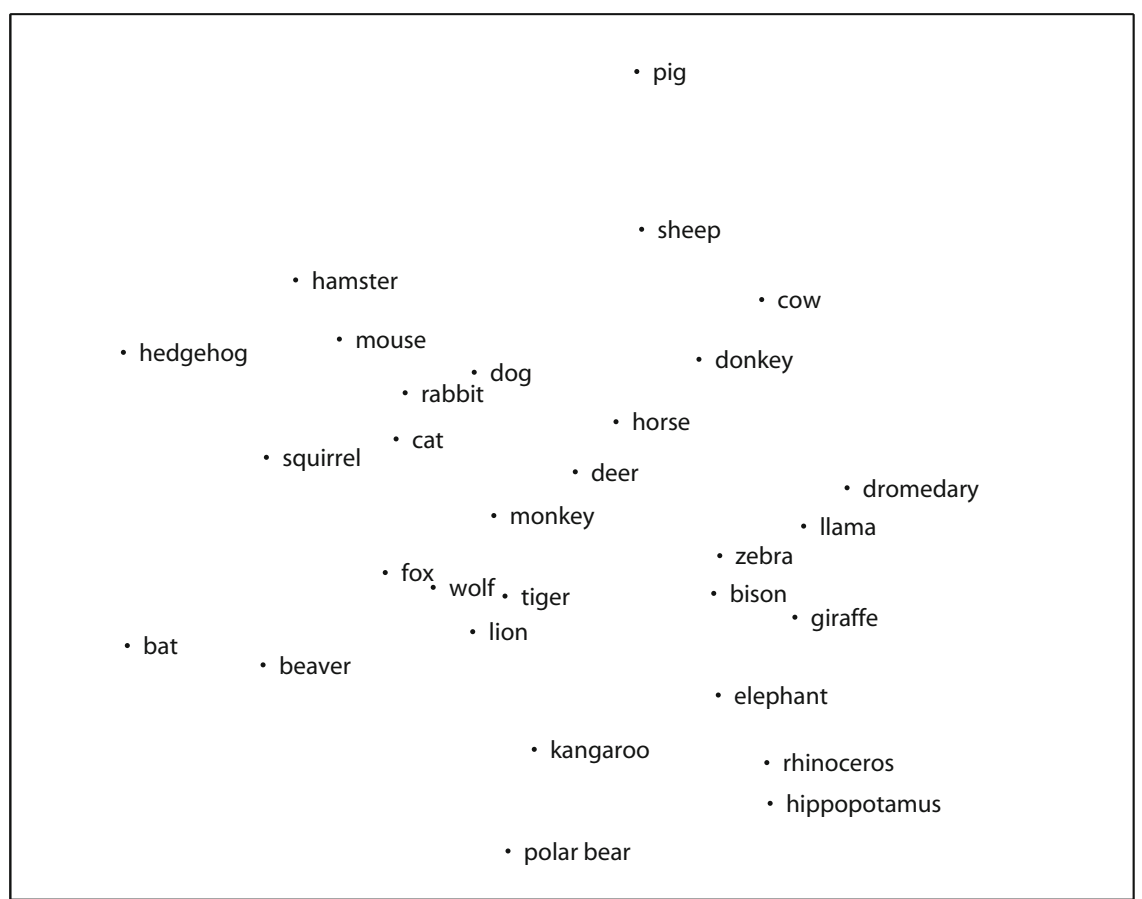

Figure 1. Example multidimensional scaling representation of the category mammals.

in the semantic verification task (e.g., Rips, Shoben, \& Smith, 1973) and the same-different task (e.g., Carramazza, Hersh, \& Torgerson, 1976), and the prediction of choice probabilities in an analogical reasoning task (e.g., Rips et al., 1973; Rumelhart \& Abrahamson, 1973). Spatial representations also play a central role in a number of models of categorization such as the generalized context model (Nosofsky, 1984, 1986), the boundary decision model (Ashby \& Gott, 1988), and prototype models (e.g., Medin \& Schwanenflugel, 1981; Smith \& Minda, 1998). Although these models were developed in the context of categorization tasks involving simple abstract or perceptual stimuli, it has recently been demonstrated that it is possible to successfully apply these classes of model to the prediction of categorization decisions using real-world semantic concepts such as fruits and vegetables, and carnivores and herbivores (e.g., Smits, Storms, Rosseel, \& De Boeck, 2002; Verbeemen, Vanpaemel, Pattyn, Storms, \& Verguts, 2007).

\section{Measuring Similarity}

As has been indicated, in order to generate spatial representational models, it is necessary to first obtain similarity data. Numerous methods have been employed to obtain empirical similarity data, including direct ratings, sorting tasks, triad ratings, conditional probability ratings, rank proximity in generation tasks, and a range of feature-applicability-based measures (see Borg \& Groenen, 2005, for a review). By far the most common method of obtaining similarity data in psychological research is the direct pairwise rating technique: A review of 120 published semantic category similarity data sets indicated that $65 \%$ of the similarity data had been obtained using a direct pairwise comparison method. The next most common method of obtaining empirical similarities was to derive them from feature matrices $(20.8 \%)$, with the other methods each making up less than $10 \%$ of the remaining data sets.

Although these data collection methods differ considerably, it is implicitly assumed that the various measures all quantify the same underlying psychological construct. However, it should be noted that very few studies have attempted to directly compare the predictions made by different similarity measures, and when comparisons have been made they have been restricted to the similarity structure of a single category (e.g., Bijmolt \& Wedel, 1995; Henley, 1969; Johnson \& Tversky, 1984; Wish, 1976).

\section{Outline}

In this study, we aim to compare the relative utility of similarity data gathered using either direct pairwise ratings or feature-based methods in regard to generating spatial representational models of 15 well-known semantic concepts. We do this in two ways.

First, we are interested in the goodness of fit between the empirical similarities and the similarity structure of the derived MDS models. The primary aim of a spatial model is to represent the similarity structure of a given concept within a multidimensional space, and the extent to which the different similarity data sets can be accurately represented by a spatial model can be taken as an indication of the suitability of a given similarity type as input data for multidimensional scaling. 
Interestingly, past studies have indicated that MDS recovers the interitem structures of different forms of similarity data with varying success. Of particular interest to the present study is the finding in Johnson and Tversky (1984) indicating that feature-based similarities were more successfully recovered by an MDS algorithm than directly rated similarities. However, the Johnson and Tversky study was limited to a single category (perceptions of risks), and only 2- and 3-dimensional representations were compared. In this study we employ 15 well-known conceptual data sets, and compare representational fits across 1 to 10 dimensions.

The second aim of this study is to compare the predictive validity of the spatial models derived from the different similarity data types. It is important to note that just because a given similarity data set can be accurately represented by a spatial model does not mean that the resulting representation is of any psychological interest. Theoretically, it would be possible to perfectly represent the similarity structure of the upper triangle of a randomly generated similarity data set, but the resulting representation would be as devoid of meaning as the data on which it was based.

A quantifiable approach to assessing the psychological validity of the representations is to compare them in regard to the prediction of independent psychological variables (Verheyen et al., 2007). For example, a widely replicated finding in the semantic concept literature is that many natural categories exhibit graded structure: Although participants may agree that a set of exemplars all belong to the same category, they tend to differentiate between the exemplars in regard to their rated typicality as members of that category (e.g., Rosch \& Mervis, 1975). Following this, in the present study we assess the validity of the representations derived from the different similarity measures in regard to their ability to predict three empirical measures of graded category structure: typicality ratings, exemplar generation frequencies, and categorization response times.

\section{METHOD}

Unless otherwise specified, all of the data in this study were taken from the normed semantic category data set reported in De Deyne et al. (2008) and De Deyne (2008). Detailed descriptions of the methods employed to collect these data can be found in these articles, and so we will restrict the following to merely describing the data.

The present study focused on 15 semantic concepts. Five of the categories were taken from the domain of animals (mammals, birds, fish, insects, and reptiles), 6 from the domain of artifacts (kitchen utensils, musical instruments, clothing, weapons, vehicles, and tools), 2 from the domain of foods (fruits and vegetables), and 2 from the domain of activities (sports and professions). Each of the categories contained between 20 and 33 exemplars. The method employed to define the category membership of the exemplars is outlined in detail in De Deyne et al. (2008) and Ruts et al. (2004).

\section{Directly Rated Similarity Data}

The directly rated similarity data were obtained using a standard pairwise similarity rating task: Participants were presented with all of the unique pairings of the exemplars within a given category and were asked to rate the similarity of each pair.

An averaged similarity matrix was obtained for each category by taking the mean of each similarity rating across the participants. Following this, each averaged similarity matrix was normalized to lie in the range of $0-1$.

\section{Feature-Based Similarity Data}

Feature-by-exemplar matrices. The feature-based similarities were derived from feature-by-exemplar matrices. Each matrix was made up of columns representing each of the exemplars within a single category, and rows representing descriptive features. The matrices contained 156 to 382 features. Each cell in a given matrix contained either a 1 or a 0 , indicating the applicability of each feature/exemplar combination. Four participants completed a separate matrix for each category.

\section{The Feature-Based Similarity Measures}

Numerous methods are available for obtaining similarities from feature-by-exemplar matrices. In this article we focus on five methods: a correlation-based measure, three feature-matching measures, and a feature distance measure.

Each of the measures was applied to each of the categories. Four separate similarity matrices were calculated for each category (i.e., one for each of the four exemplar-byfeature matrices). An averaged similarity matrix was calculated for each category by taking the mean of the four similarity matrices. Each averaged matrix was normalized to lie in the range of $0-1$.

Feature vector correlation. A common approach to deriving a measure of similarity between two exemplars is to calculate the correlation between the feature vectors associated with each stimulus. Given the feature vectors $v_{i}$ and $v_{j}$, the similarity between exemplars $i$ and $j$ is given by the correlation measure:

$$
s_{i j}=\frac{\sum_{k}\left(v_{i k}-\bar{v}_{i}\right)\left(v_{j k}-\bar{v}_{j}\right)}{\left[\sum_{k}\left(v_{i k}-\bar{v}_{i}\right)^{2} \sum_{k}\left(v_{j k}-\bar{v}_{j}\right)^{2}\right]^{\frac{1}{2}}},
$$

where $k$ indicates the number of features in each vector.

Feature matching. An alternative approach to conceptualizing the similarity between two exemplars relates to their degree of featural overlap. Numerous methods have been proposed to account for interexemplar similarity on the basis of common features, distinctive features, or a combination of the two (see Tversky, 1977, for a review). In this article we employ three similarity measures based on the contrast model (Tversky, 1977). The contrast model generalizes a number of feature-matching measures by employing a parameter that controls the relative contribution of common and distinctive features to 
the overall similarity measure. Following Navarro and Lee (2004), interitem similarity under the contrast model can be calculated as

$$
\begin{aligned}
s_{i j}= & \lambda+\left[\rho \sum_{k} v_{i k} v_{j k}\right]-\left[(1-\rho) \sum_{k} v_{i k}\left(1-v_{j k}\right)\right] \\
& -\left[(1-\rho) \sum_{k}\left(1-v_{i k}\right) v_{j k}\right],
\end{aligned}
$$

where $0 \leq \rho \leq 1$, and $\lambda$ is an additive constant indicating a universal level of interexemplar similarity. From this it can be seen that setting the parameter $\rho$ to low values emphasizes distinctive features, whereas setting $\rho$ to high values emphasizes common features. In this study we employed three values of $\rho(0,1$, and .5) corresponding (respectively) to a purely distinctive feature contrast, a purely common feature contrast, and an equally weighted common and distinctive feature contrast.

Feature distance. According to this approach, each row in the feature-by-exemplar matrix can be conceptualized as a dimension in a multidimensional space, with the location of each exemplar along each dimension in this space indicated by the presence or absence of the corresponding feature. Following this, it is possible to calculate the similarity between any two exemplars in the feature space using

$$
s_{i j}=\lambda-\left[\sum_{k}\left|v_{i k}-v_{j k}\right|^{r}\right]^{\frac{1}{r}},
$$

where $\lambda$ is a constant used to transform the distances into similarities, and the Minkowski $r$ metric is set to 2, resulting in Euclidean distances. It should be noted that we did not implement a feature distance measure using a city block metric (i.e., $r=1$ ) because this produces similarity data identical to the distinctive features version of the feature-matching measure described above.

\section{Spatial Representations}

Spatial representations were generated for each of the similarity data sets using the metric multidimensional scaling algorithm described in Lee (2001). ${ }^{1}$ The representations were generated with 1 to 10 dimensions for each of the 15 categories using both a Euclidean and a city block metric. Because the results of the subsequent analyses were almost identical regardless of the underlying metric, we will only report the analyses for representations based on a Euclidean metric.

\section{Empirical Measures of \\ Graded Category Structure}

In order to test the predictive validity of the spatial representations generated from the different similarity data types, we employed three empirical measures of graded category structure: typicality ratings, exemplar generation frequencies, and categorization response times. The typicality and exemplar generation frequency data were taken from De Deyne et al. (2008), and the categorization response time data from De Deyne (2008).

\section{ANALYSES}

In the following, we compare the relative utility of the six different similarity data types (five feature-derived similarity types and the direct similarity ratings). We will first explore the degree of intercorrelation between the similarities, and compare their associated data precision and reliability. Following this we will address the two main aims of our study by comparing the similarities in regard to representational goodness of fit and predictive validity.

For the remainder of this article we will refer to the different similarity data types using the following: directrating (direct pairwise similarity ratings), $F$-correlation (feature vector correlation), $F$-distance (Euclidean feature distance), $F$-distinctive (distinctive feature contrast), $F-C \& D$ (equal-weighted common and distinctive feature contrast), and $F$-common (common feature contrast).

\section{Intercorrelation, Data Precision, and Interrater Reliability}

Given that it is implicitly assumed that the different similarity measures are all quantifying the same underlying psychological construct, it is worthwhile to examine the degree of intercorrelation among the measures. For each similarity data type in each category, we obtained a vector of similarity values from the upper triangle of each similarity matrix. Table 1 shows the intercorrelation between these similarity vectors considered across all 15 categories. All of the correlations were highly significant $(p<.001, N=5,723)$.

A number of interesting patterns emerge from these results. First, the strongest correlation with the direct-rating data is found for $\mathrm{F}-\mathrm{C} \& \mathrm{D}$, supporting the results of a previous study (Tversky, 1977) in which the best feature-based predictor of directly rated similarities was found to be a linear contrast of both common and distinctive features. It is therefore not surprising that the next best predictor of the direct-rating similarities in the present study was $\mathrm{F}$-correlation, because feature vector correlations also employ both common and distinctive feature information.

Second, the common-feature-based measure appears to produce reasonably idiosyncratic similarity data: Specifically, the correlations between $\mathrm{F}$-common similarities and the other feature-based similarities are much weaker than the correlations between the remaining feature-based

Table 1

Intercorrelations Among the Six Similarity Data Types Across the 15 Semantic Categories, and Mean Data Precision $(\bar{\sigma})$ and Interrater Reliability $(\bar{r})$

\begin{tabular}{lccccccccc}
\hline & \multicolumn{7}{c}{ and Interrater Reliability $(\boldsymbol{r})$} \\
\cline { 2 - 7 } & 1 & 2 & 3 & 4 & 5 & 6 & $\bar{\sigma}$ & $\bar{r}$ \\
\hline 1. Direct-rating & - & .67 & .61 & .60 & .68 & .61 & .19 & .89 \\
2. F-correlation & & - & .92 & .92 & .98 & .81 & .07 & .91 \\
3. F-distance & & & - & .99 & .92 & .59 & .05 & .90 \\
4. F-distinctive & & & & - & .93 & .58 & .08 & .90 \\
5. F-C\&D & & & & & - & .82 & .06 & .90 \\
6. F-common & & & & & & - & .08 & .90 \\
\hline
\end{tabular}


similarities. The importance of this distinction will be made clear in light of the goodness of fit and predictive validity analyses in the following sections.

Table 1 also displays the data precision $(\bar{\sigma})$ and interrater reliability $(\bar{r})$ of the six similarity types averaged over the 15 categories. We measured interrater reliability using averaged Spearman-Brown corrected split-half correlations calculated over 10,000 random splits of the similarity matrices corresponding to each participant. Following Lee (2001), we measured data precision by calculating the standard deviation of the value in each cell in the upper triangle of the matrix corresponding to each participant, and then finding the average standard deviation for the category. These two measures provide important indications of the degree of homogeneity or agreement in the responses of the participants. The $\bar{r}$ value provides an indication of the degree of agreement across participants regarding the ordering of the similarity values. The $\bar{\sigma}$ value, on the other hand, provides an indication of the degree of agreement across participants regarding the absolute magnitude of the similarity values. It should be noted that these measures dissociate from each other: It is possible for a data set to have high reliability and poor precision, and vice versa. Ideally, if the participants are responding in a homogeneous fashion, a data set should have a high $\bar{r}$ and a low $\bar{\sigma}$.

With regard to reliability, the $\bar{r}$ values in Table 1 indicate a high degree of interrater reliability for all of the similarity data types (any $\bar{r}$ value of greater than .80 can be safely interpreted as an indication of high interrater agreement). With regard to data precision, Lee (1999) suggests that for
$0-1$ normalized similarity matrices, $\bar{\sigma}$ values of $\leq .10$ correspond to reasonably precise data, whereas values of $\geq .20$ indicate reasonably imprecise data. It is interesting to note that all of the feature-based similarity types have $\bar{\sigma}$ values of $\leq .10$. However, the direct-rating $\bar{\sigma}$ value is markedly worse than all of the other similarity data types. ${ }^{2}$

\section{Representational Goodness of Fit}

As was indicated in the introduction, the primary purpose of a spatial model is to represent the similarity structure of a given category within a multidimensional space. It is therefore very important that there is a high degree of correspondence between the empirical similarities and the similarity structure of the representational model. We measure this representational goodness of fit as the proportion of empirical similarity $\left(s_{i j}\right)$ variance accounted for (VAF) by the model similarity predictions $\left(\hat{s}_{i j}\right)$ :

$$
\mathrm{VAF}=1-\frac{\sum_{i<j}\left(s_{i j}-\hat{s}_{i j}\right)^{2}}{\sum_{i<j}\left(s_{i j}-\bar{s}\right)^{2}},
$$

where $\bar{s}$ is the average of the empirical similarities.

We compared the fit between each similarity data set and its resulting representation to determine whether there are differences between the various similarity types in regard to the adequacy of their representational fit across different levels of dimensional complexity. Figure 2 shows the representational fits for the six similarity data types using

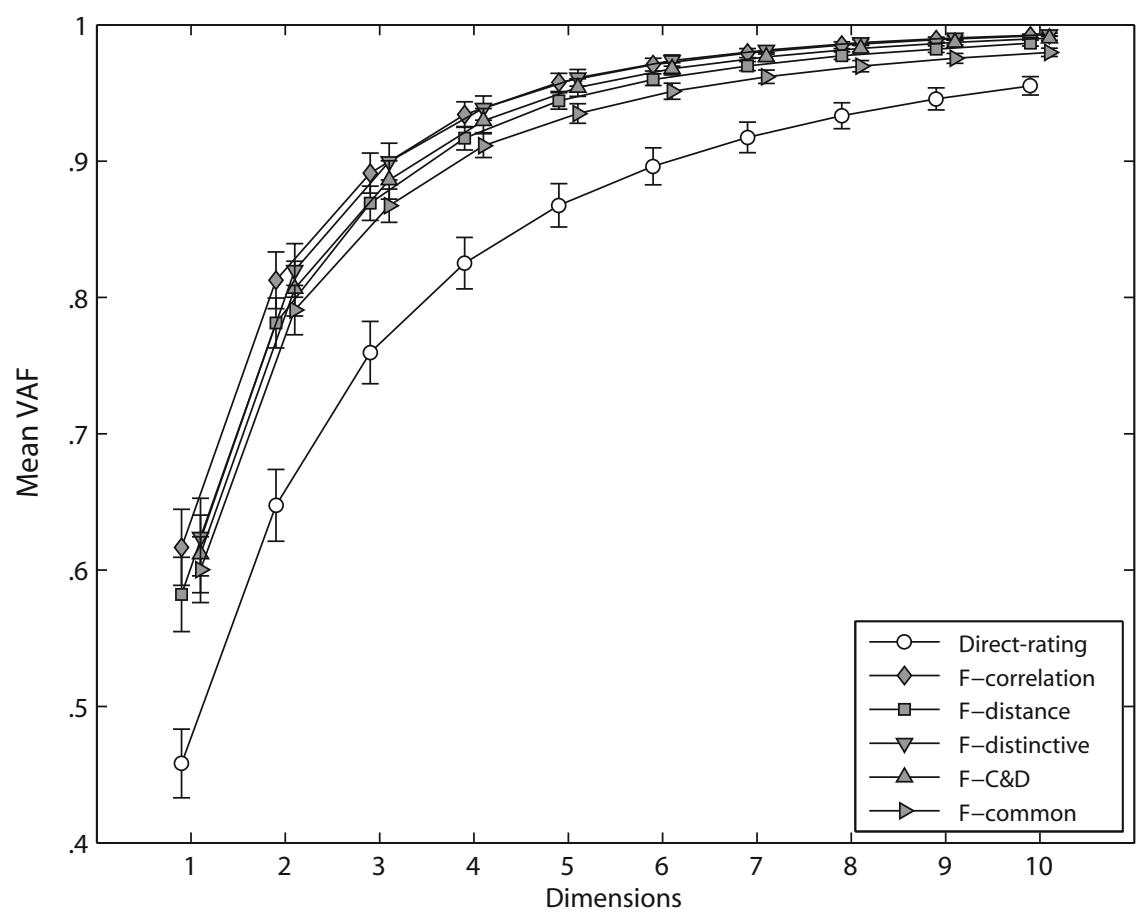

Figure 2. Mean proportion of empirical similarity $\left(s_{i j}\right)$ variance accounted for (VAF) by the model similarity predictions $\left(\hat{s}_{i j}\right)$ using spatial models with 1 to 10 dimensions. The VAF values were averaged across the 15 semantic categories. 
Table 2

Mean Skewness and Proportion of Elongated Triangles, Averaged Across the 15 Categories

\begin{tabular}{lcccccc}
\hline & Direct-Rating & F-Correlation & F-Distance & F-Distinctive & F-C\&D & F-Common \\
\hline Skewness & -1.41 & -0.52 & -0.82 & -0.36 & -0.53 & -0.67 \\
Elongation & .70 & .66 & .66 & .65 & .66 & .67 \\
\hline
\end{tabular}

spatial representations with 1 through 10 dimensions. Because of the difficulties of presenting the results for each category separately, we use in Figure 2 a summary plot showing the VAF averaged across the 15 categories.

As can be seen, regardless of the type of similarity data that the spatial models were generated from, the representational fits all improve as the dimensionality of the representation increases from 1 to 10 dimensions. However, there is a striking difference between the quality of the fits for the direct-rating and feature-based similarity data sets, with the direct-rating representations providing consistently worse fits, regardless of the assumed underlying dimensionality.

Somewhat surprisingly, the 10-dimensional directrating representations only manage to achieve the same level of fit as is achieved by the feature-based representations using around half the number of dimensions. Given that in multidimensional scaling the number of free parameters equals the number of exemplars multiplied by the number of dimensions, this difference in parametric complexity can be considered nontrivial.

It is important to note that the superiority of the goodness of fit for the feature-based representations was also found when each category was considered in isolation. Although the ordering of the fits for the feature-based representations showed some variability across categories, almost without exception the fits of the direct-rating representations were consistently poorer than those of all of the feature-based representations.

It is not immediately obvious why there should be such a large difference in the representational fits of the different similarities. One potential explanation can be found in the distributional properties of the similarity data. For example, simulation studies (Pruzansky, Tversky, \& Carroll, 1982) have shown that spatial representations tend to produce positively skewed distributions of interexemplar distances. Another potentially useful diagnostic distributional property is proportion of elongated triangles: the distances between any three exemplars in a representation form a triangle with a short, middle, and long side. If the middle side is closer in length to the long side than the short side, the triangle is elongated. Pruzansky et al. reasoned that spatial representations should have a lower proportion of elongated triangles compared with alternative representational models such as additive trees.

Table 2 shows the mean skewness and proportion of elongated triangles in the 1-s empirical proximity data. As before, the values were calculated by averaging across the 15 semantic categories. The data show a small degree of variation in the elongation values across the feature-based proximities. Importantly, however, all of the feature-based proximities have a lower proportion of elongated triangles than the direct-rating proximities. The skewness data are even more revealing: The direct-rating proximities are far more negatively skewed than the feature-based proximities. Taken together, these diagnostic properties provide a reasonable explanation for the pattern of results found in the goodness-of-fit analyses.

The skewness and elongation values also suggest that the direct-rating similarities might be more adequately fit by an additive tree representation than by a spatial representation. In order to determine whether the poor representational fit of the direct-rating data was due to the form of the representational model, we generated additive tree representations using the algorithm described in Lee (1999) for the 15 categories, using 1 to 20 internal nodes.

Surprisingly, the results indicated that the direct-rating tree representations were actually worse than any of the feature-based representations. In general, however, the quality of the tree fits was much poorer than with those obtained using spatial representations, with the difference between the best spatial representation generated from each similarity data type and the corresponding best tree representation being on average .15 .

\section{Predictive Validity}

It has been suggested that the validity of a spatial model can be assessed in terms of the representation's interpretability. A classic example of this can be found in the representations of the category mammals in Henley (1969) and Rips et al. (1973), in which the dimensions are interpreted as "size" and "predacity/ferocity." Nonetheless, many spatial representations of semantic concepts are far less interpretable, and yet appear to be able to adequately represent the empirical similarity structure of the category. Furthermore, the difficulty of attempting to make qualitative interpretations of the 900 spatial representations $(6$ similarity types $\times 15$ categories $\times 10$ dimensionalities) under consideration in the present study precluded this form of assessment.

An alternative, quantifiable approach to assessing the validity of the representations is to compare them in terms of their ability to predict external empirical variables. For example, Ameel and Storms (2006) and Verheyen et al. (2007) demonstrated that empirical typicality ratings of category exemplars could be successfully predicted by the distance between each exemplar within a multidimensional category space and the category prototype or centroid. Specifically, highly typical exemplars tended to be located close to the category centroid, whereas atypical exemplars tended to be located closer to the edge of the category boundary. Following this approach, we assessed the validity of the representa- 


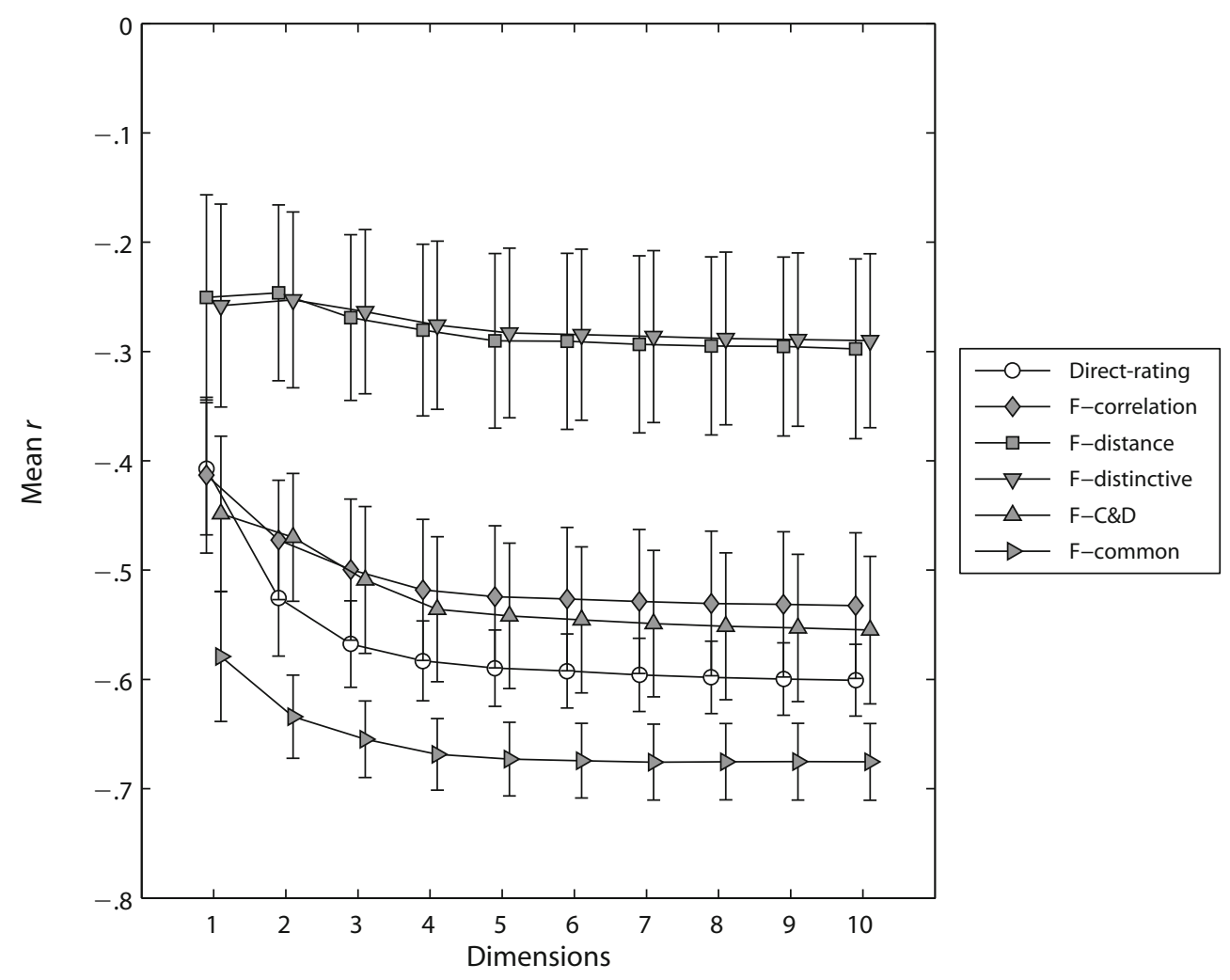

Figure 3. Correlation ( $r$ ) between empirical typicality rating and distance-from-centroid for spatial representations generated from the six similarity data sets using 1 to 10 dimensions. The correlation values are averaged over the 15 categories. Error bars indicate one standard error.

tions generated from the different similarity data types in terms of their ability to predict empirical typicality ratings, and two additional empirical measures of graded category structure: exemplar generation frequency and categorization response time.

We modeled these empirical variables as the distance between each exemplar within a category space and the category centroid (where the centroid was the average of the exemplar locations along each dimension). ${ }^{3}$ We expected to find a negative relationship between distancefrom-centroid and the variables' typicality and exemplar generation frequency, and a positive relationship between distance-from-centroid and categorization response time. The results of the analyses, averaged across the 15 categories, are shown in Figures 3 through 5.

First, as was found in Verheyen et al. (2007), in the majority of cases the quality of the predictions tended to show a slight increase as the dimensionality of the solution increased. This can be seen most clearly in the typicality predictions (Figure 3 ), and is particularly noticeable in the direct-rating predictions across all three empirical variables. It should be noted, however, that in some cases the slope is close to zero, or even shows an increase in fit in the wrong direction as dimensionality increases (such as the $\mathrm{F}$-distance and $\mathrm{F}$-distinctive predictions of exemplar generation frequency and categorization response time; Figures 4 and 5, respectively).
Second, there is a noticeable difference in the quality of the predictive fits across the three variables. The best predictions for typicality reach mean values of around -.67, whereas the best predictions for exemplar generation frequency and categorization response time are -.50 and .42 , respectively. Such a result is unsurprising, given that typicality is generally assumed to be a stronger measure of category centrality than the other two measures.

Third, there are noticeable differences in the quality of the predictive fits across the different similarity types. The $\mathrm{F}$-correlation, $\mathrm{F}$-distance, and $\mathrm{F}$-distinctive predictions are consistently poor, and in the cases of exemplar generation frequency and categorization response time, close to zero. In contrast, the best predictors for all three empirical variables were the common-features-based similarities (F-common), which consistently outperformed the other similarity measures. Furthermore, there is a noticeable difference between the quality of fits for representations based on these two similarity types, and the next best performing similarity type (i.e., direct-rating).

Correlation coefficients are useful in that they provide an easily interpretable measure of predictive fit. However, it could be argued that they are not well suited to distinguishing between different models: a correlation of -.57 is stronger than a correlation of -.55 , but it is difficult to determine whether this difference is of any psychological interest. In light of this, we employ the Bayesian infor- 


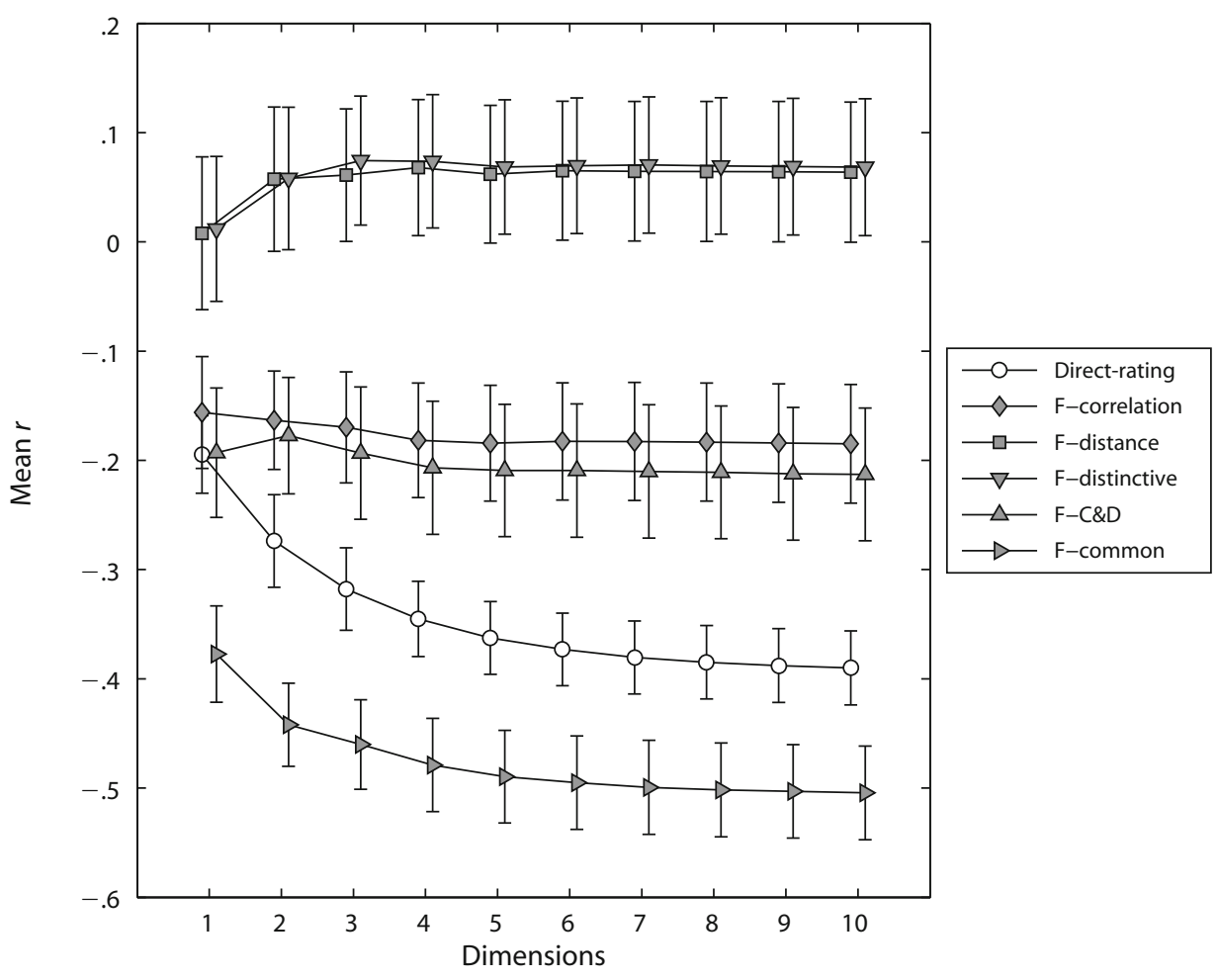

Figure 4. Correlation $(r)$ between empirical exemplar generation frequency and distance-fromcentroid for spatial representations generated from the six similarity data sets using 1 to 10 dimensions. The correlation values are averaged over the 15 categories. Error bars indicate one standard error.

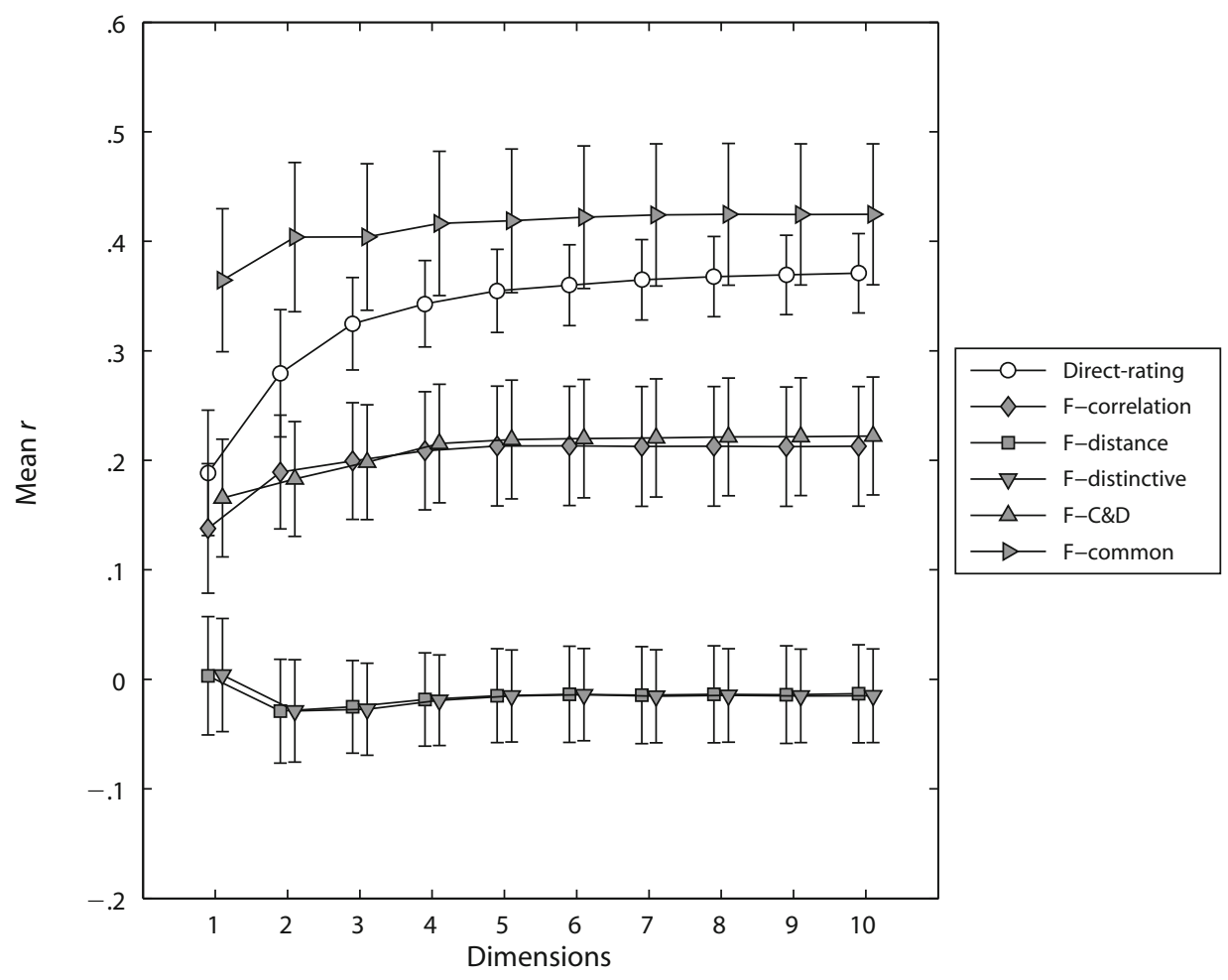

Figure 5. Correlation $(r)$ between empirical categorization response time and distance-from-centroid for spatial representations generated from the six similarity data sets using 1 to 10 dimensions. The correlation values are averaged over the 15 categories. Error bars indicate one standard error. 
Table 3

Bayes Factors for the Predictive Fits of the Two Most Likely Representational Models Across 1 to 10 Dimensions

\begin{tabular}{|c|c|c|c|c|c|c|c|c|c|c|}
\hline & \multicolumn{10}{|c|}{ Dimensions } \\
\hline & 1 & 2 & 3 & 4 & 5 & 6 & 7 & 8 & 9 & 10 \\
\hline \multicolumn{11}{|c|}{ Typicality } \\
\hline Direct-rating & $5.38 \times 10^{9}$ & $6.89 \times 10^{5}$ & $6.57 \times 10^{5}$ & $4.15 \times 10^{5}$ & $5.40 \times 10^{5}$ & $5.97 \times 10^{5}$ & $6.20 \times 10^{5}$ & $4.77 \times 10^{5}$ & $3.92 \times 10^{5}$ & $3.63 \times 10^{5}$ \\
\hline $\mathrm{F}$-common & 1.00 & 1.00 & 1.00 & 1.00 & 1.00 & 1.00 & 1.00 & 1.00 & 1.00 & 1.00 \\
\hline \multicolumn{11}{|c|}{ Exemplar Generation Frequency } \\
\hline Direct-rating & $4.52 \times 10^{7}$ & $2.81 \times 10^{8}$ & $4.80 \times 10^{8}$ & $1.12 \times 10^{9}$ & $9.25 \times 10^{8}$ & $8.13 \times 10^{8}$ & $6.59 \times 10^{8}$ & $5.44 \times 10^{8}$ & $4.55 \times 10^{8}$ & $4.33 \times 10^{8}$ \\
\hline $\mathrm{F}$-common & 1.00 & 1.00 & 1.00 & 1.00 & 1.00 & 1.00 & 1.00 & 1.00 & 1.00 & 1.00 \\
\hline \multicolumn{11}{|c|}{ Categorization Response Time } \\
\hline Direct-rating & 85.34 & 118.02 & 100.00 & 82.42 & 67.25 & 65.39 & 61.61 & 58.81 & 54.42 & 52.67 \\
\hline $\mathrm{F}$-common & 1.00 & 1.00 & 1.00 & 1.00 & 1.00 & 1.00 & 1.00 & 1.00 & 1.00 & 1.00 \\
\hline
\end{tabular}

Note-Bayes factors are given in relation to the most likely model (i.e., B.f. $=1.00$ ).

mation criterion (Schwarz, 1978) to compare the relative likelihoods of the predictions made using the different spatial representations.

Following this approach, we calculate the weighted sum-square-error fit of the empirical variable $(e)$ and representation-based prediction as

$$
\mathrm{WSSE}=\sum_{i} \frac{1}{\sigma_{i}^{2}}\left(e_{i}-\alpha+\beta \eta_{i}\right)^{2}
$$

where $\sigma^{2}$ is the variance of the empirical variable, and $\alpha$ and $\beta$ are regression coefficients employed to linearly transform the distance $(\eta)$ between each exemplar and the category centroid into a prediction on the same scale as the empirical variable. Under the assumption of a Gaussian likelihood function, the Bayesian information criterion (BIC) is calculated as

$$
\mathrm{BIC}=\mathrm{WSSE}+p \ln n,
$$

where $p$ is the number of free parameters (which in this case is equal to the number of regression coefficients), and $n$ is the number of category exemplars. The relative likelihood of the data fits can then be compared using Bayes factors (Kass \& Raftery, 1995).

Using the WSSE fits across all 15 categories, we calculated a single BIC value for the predictions of the representations based on the six different similarity types using dimensionalities of 1 to 10 . Table 3 shows the Bayes factors for the predictive fits to empirical typicality, exemplar generation frequency, and categorization response time

Table 4

Bayes Factors for Predictions Based on the Different Similarity Types' Optimal Representational Model in Each Category

\begin{tabular}{lllc}
\hline & & $\begin{array}{c}\text { Exemplar } \\
\text { Generation } \\
\text { Frequency }\end{array}$ & $\begin{array}{c}\text { Categorization } \\
\text { Response } \\
\text { Time }\end{array}$ \\
\hline Direct-rating & $2.44 \times 10^{4}$ & $2.13 \times 10^{8}$ & 49.92 \\
F-correlation & $1.44 \times 10^{4}$ & $3.63 \times 10^{12}$ & 245.70 \\
F-distance & $1.76 \times 10^{10}$ & $2.11 \times 10^{12}$ & $1.86 \times 10^{3}$ \\
F-distinctive & $1.27 \times 10^{11}$ & $4.13 \times 10^{12}$ & $2.21 \times 10^{3}$ \\
F-C\&D & $1.69 \times 10^{3}$ & $7.66 \times 10^{9}$ & 218.95 \\
F-common & 1.00 & 1.00 & 1.00 \\
\hline
\end{tabular}

Note-Bayes factors are given in relation to the most likely model (i.e., B.f. $=1.00)$ of the two most likely spatial representational models: $\mathrm{F}-$ common and direct-rating.

Using Jeffreys's (1961) guidelines for interpreting Bayes factors (where Bayes factors of $<3.2,3.2$ to 10,10 to 100 , and $>100$ can be interpreted as "not worth more than a bare mention," "substantial" evidence, "strong" evidence, and "decisive" evidence, respectively), we can see that for all three criterion variables, the fits of the direct-rating representation predictions are well over the criterion for a strong difference in relative likelihood. ${ }^{4}$

Verheyen et al. (2007) demonstrated that external empirical variables such as typicality can be used as a means of determining the optimal dimensionality of a spatial representation. Following this approach, we could assume that for each similarity data type the representational dimensionality that maximizes the likelihood of the predictive fit for a given category is an indication of the optimal representational dimensionality for the category. If we take this to be true, comparing the predictions across fixed dimensionalities (such as is the case in Table 3) might potentially unfairly penalize the models because the comparisons would include suboptimal representations. In light of this potential criticism, we also conducted Bayesian analyses using only the optimal representations of each similarity data type in each category. The results of the analyses are reported in Table 4. As can be seen, once again the representations based on $\mathrm{F}$-common similarities provide the most likely predictions of all three empirical variables.

\section{DISCUSSION}

The main aim of this study was to assess the utility of different forms of empirical similarity data for generating spatial representations of well-known semantic concepts. We assessed utility in two ways: representational goodness of fit and predictive validity. We also assessed the degree to which the different similarity data types were intercorrelated, and compared their mean reliability and precision. The results of our analyses suggest that there are nontrivial differences between the similarity data types under comparison in this study.

In regard to representational goodness of fit, the results suggest that the feature-based similarities were more ad- 
equately represented by a spatial model than by directly rated similarities. As could be seen in Figure 2, the difference between the mean VAF of the direct-rating and feature-based similarities was readily discernible, with the direct-rating representations requiring around twice the number of dimensions to obtain the same level of fit as the feature-based representations. Furthermore, this pattern of results held regardless of assumptions made about the underlying dimensionality of the representations, or the metric (Euclidean vs. city block) employed to generate the representations.

As was discussed, this difference in representational goodness of fit appeared to be due to differences in the distributional properties of the pairwise and feature-based similarity data. Specifically, the direct-rating proximity data had a higher mean proportion of elongated triangles, and a stronger mean negative skew than the feature-based proximities, suggesting that the direct-rating data were less suited to spatial representation. Surprisingly, the direct-rating tree representations also proved to be notably less adequate than the tree representations derived from feature-based similarities.

The skewness data are also interesting because they tell us something about the tasks employed to generate the different similarity data sets. On the one hand, the direct pairwise rating task appears to emphasize the high latent similarity between the exemplars as members of the same category (relative to nonmembers), resulting in many high similarity ratings and few low similarity ratings. On the other hand, the feature applicability task forces participants to consider the exemplars in light of a wide range of descriptive features (some of which may only apply to a small subset of the category exemplars). As a result, the feature applicability task appears to be more successful than the direct rating task at differentiating between the exemplars with regard to within-category similarity structure.

A further note should be made here regarding our additional analyses involving the goodness of fit for additive tree representations. We found that all of the similarity data types were more adequately represented by spatial representations than by additive tree representations. This is an important finding, given that it has been generally accepted (on the basis of evidence reported in Pruzansky et al., 1982) that semantic concept data are better represented by additive tree representations than by spatial representations. Investigating the reason for this difference lies outside of the context of this article, but it is possible (as has been suggested by Shoben \& Ross, 1987) that it is due to the fact that the categories employed in Pruzansky et al. included both category exemplars and the category label. It would be interesting to explore this further in a future study.

With regard to the second assessment criterion, predictive validity, the results indicated a wide variation in the utility of the spatial representations generated from the different similarity types. Notably, the representations derived from a number of the feature-based measures (Euclidean feature distance, feature vector correlation, and distinctive feature contrast) produced consistently poor predictions of all three empirical variables regardless of the underlying dimensionality of the representation. Although in Table 3 we only presented the Bayes factors for the two most likely representational models, we can infer from these results that the representations based on the remaining similarity types made predictions that are highly unlikely (i.e., at least $3.63 \times 10^{5}, 4.52 \times 10^{7}$, and 52.67 times less likely than the most likely representation for the typicality, exemplar generation frequency, and response time predictions, respectively). This is an important finding, given that in past studies the most common methods of obtaining similarities from feature data have been either feature vector correlation or some form of feature distance measure (recall that the $\mathrm{F}$-distinctive similarities are equivalent to similarities derived from city block feature distances). Similarly, the representations based on the predominant measure of similarity in the literature (direct pairwise ratings) were also demonstrated to be suboptimal in comparison with the $\mathrm{F}-$ common representations.

The finding that the empirical measures of graded category structure were best accounted for using representations derived from similarity based on feature commonality accords with the results of previous studies that have employed the family resemblance (Rosch \& Mervis, 1975) and polymorphous concept (Hampton, 1979) models to predict graded category structure: Typical exemplars tend to have many features in common with other category members, and atypical exemplars tend to have few features in common with other category members. It also appears that the exemplars with many features in common with other category members are generated more frequently in response to the category name and are verified as category members more quickly than are exemplars with few common features. What distinguishes the present study is that the correspondence between featural overlap and typicality has not been measured using raw features, but with a spatial representation derived from common features similarity data.

\section{Conclusions}

The results of the present study suggest that featurederived similarities are to be preferred to directly rated similarities with regard to the goodness of fit of spatial representations. This result is somewhat surprising, given the predominance of the direct pairwise rating task for collecting similarity data. In psychology, it is generally assumed that the direct rating task is the "gold standard" for the collection of similarity data. This study suggests that with regard to the similarity structure of semantic concepts, this standard needs to be revised. Whether or not the same conclusion applies to the similarity structure of perceptual and abstract stimuli is a question that remains to be tested empirically.

Furthermore, the common-features-based similarities are to be preferred to the alternative feature-based similarities with regard to the predictive validity of the representations. This finding has implications not only for researchers interested in generating spatial representations of semantic concepts, but is also potentially revealing with regard to the nature of the cognitive processes underly- 
ing similarity: Specifically, it would appear that the interexemplar similarity structure of semantic categories is a linear function of featural overlap.

The results of this study suggest that researchers interested in generating spatial representations of psychological concepts should seriously consider discarding the popular pairwise similarity rating technique in favor of similarities derived from feature-by-exemplar matrices. One of the advantages of this approach is that it allows researchers to generate the similarities using a variety of approaches. In this study, the common-features-based measure was demonstrated to be superior to the alternative feature-based measures, but it is possible that in different data sets this might not be the case. We strongly advocate that researchers employ the methods outlined in this article to determine the optimal form of the similarity data employed in their research.

Finally, given the importance of similarity within cognitive research, the considerable differences between the measures compared in this study are somewhat surprising, and suggest that we still have only a very basic understanding of this fundamental psychological construct. It would seem imperative to focus on improving our understanding of similarity in future studies.

\section{AUTHOR NOTE}

This research was partly completed while the authors were visiting research fellows at the University of Adelaide. We thank Simon De Deyne for providing us with his categorization response time data, and Steven Verheyen and Dan Navarro for their helpful comments and insight. This research was partly supported by Grants OT/05/24 and IDO/02004 from the Research Fund Katholieke Universiteit Leuven. Correspondence related to this article may be sent to M. J. Dry, Department of Psychology, Tiensestraat 102, B3000 Leuven, Belgium (e-mail: matt.dry@psy.kuleuven.be).

\section{REFERENCES}

Ameel, E., \& Storms, G. (2006). From prototypes to caricatures: Geometric models for concept typicality. Journal of Memory \& Language, 55, 402-421.

Ashby, F. G., \& GotT, R. E. (1988). Decision rules in the perception and categorization of multidimensional stimuli. Journal of Experimental Psychology: Learning, Memory, \& Cognition, 14, 33-53.

Ashmore, R. D., GrifFo, R., \& Green, R. (2007). Dimensions and categories underlying thinking about college student types. Journal of Applied Social Psychology, 37, 2922-2950.

Bijmolt, T. H. A., \& WedEL, M. (1995). The effects of alternative methods of collecting similarity data for multidimensional scaling. International Journal of Research in Marketing, 12, 363-371.

BorG, I., \& Groenen, P. J. F. (2005). Modern multidimensional scaling: Theory and applications. New York: Springer.

Boverie, P. E., Scheufele, D. J., \& RaYmond, E. L. (1994). Multimethodological approach to examining risk-taking. Current Psychology, 13, 289-302.

Carramazza, A., Hersh, H., \& Torgerson, W. S. (1976). Subjective structures and operations in semantic memory. Journal of Verbal Learning \& Verbal Behavior, 15, 103-117.

Clark, H. H. (1968). On the meaning and use of prepositions. Journal of Verbal Learning \& Verbal Behavior, 7, 421-431.

De Deyne, S. (2008). Semantic proximity in high dimensional spaces. Unpublished doctoral dissertation, Katholieke Universiteit Leuven, Belgium.

De Deyne, S., Verheyen, S., Ameel, E., Vanpaemel, W., Dry, M. J., Voorspoels, W., \& Storms, G. (2008). Exemplar by feature applicability matrices and other Dutch normative data for semantic concepts. Behavior Research Methods, 40, 1030-1048.
ELEVÅG, B., \& STORMs, G. (2003). Scaling and clustering in the study of semantic disruptions in patients with schizophrenia: A re-evaluation. Schizophrenia Research, 63, 237-246.

Fillenbaum, S., \& RapPaPORT, A. (1971). Structures in the subjective lexicon. New York: Academic Press.

Goodwin, R., Kwiatkowska, A., Realo, A., Kozlova, A., Luu, L., \& Nizharadze, G. (2004). Social representations of HIV/AIDS in five Central European and Eastern European countries: A multidimensional analysis. AIDS Care: Psychological \& Socio-Medical Aspects of AIDS/HIV, 16, 669-680.

HAMPTON, J. A. (1979). Polymorphous concepts in semantic memory. Journal of Verbal Learning \& Verbal Behavior, 18, 441-461.

Henley, N. M. (1969). A psychological study of the semantics of animal terms. Journal of Verbal Learning \& Verbal Behavior, 8, 176-184.

JEFFREYs, H. (1961). Theory of probability. Oxford: Oxford University Press.

JoHnSON, E. J., \& TVERSKY, A. (1984). Representations of risks. Journal of Experimental Psychology: General, 113, 55-70.

KASS, R. E., \& RAFTERY, A. E. (1995). Bayes factors. Journal of the American Statistical Association, 90, 773-796.

KruSKaL, J. B. (1964a). Multidimensional scaling by optimizing goodness of fit to a nonmetric hypothesis. Psychometrika, 29, 1-27.

Kruskal, J. B. (1964b). Nonmetric multidimensional scaling: A numerical method. Psychometrika, 29, 115-129.

LEE, M. D. (1999). Algorithms for representing similarity data (Tech. Rep. No. DSTO-RR-0152). Edinburgh, South Australia: DSTO Electronics and Surveillance Research Laboratory.

LEE, M. D. (2001). Determining the dimensionality of multidimensional scaling representations for cognitive modeling. Journal of Mathematical Psychology, 45, 149-166.

McCallum, D. M., McCallum, R., \& Gurwitch, R. H. (1987). Dimensions of interpersonal events: Reward value and information value. Journal of Experimental Social Psychology, 23, 316-330.

Medin, D. L., \& Schwanenflugel, P. J. (1981). Linear separability in classification learning. Journal of Experimental Psychology: Human Learning \& Memory, 7, 355-368.

Miller, K., \& Gelman, R. (1983). The child's representation of number: A multidimensional scaling approach. Child Development, 54, 1470-1479.

Navarro, D. J., \& Lee, M. D. (2004). Common and distinctive features in stimulus similarity: A modified version of the contrast model. Psychonomic Bulletin \& Review, 11, 961-974.

Nosofsky, R. M. (1984). Choice, similarity, and the context theory of classification. Journal of Experimental Psychology: Learning, Memory, \& Cognition, 10, 104-114.

NosofSKy, R. M. (1986). Attention, similarity, and the identificationcategorization relationship. Journal of Experimental Psychology: General, 115, 39-61.

NosOFSKY, R. M. (1988). Exemplar-based accounts of relations between classification, recognition, and typicality. Journal of Experimental Psychology: Learning, Memory, \& Cognition, 14, 700-708.

Prescott, T. J., Newton, L. D., Mir, N. U., Woodruff, P. W. R., \& PARKs, R. W. (2006). A new dissimilarity measure for finding semantic structure in category fluency data with implications for understanding memory organization in schizophrenia. Neuropsychology, 20, 685-699.

Pruzansky, S., Tversky, A., \& Carroll, J. D. (1982). Spatial versus tree representations of similarity data. Psychometrika, 47, 3-24.

Rips, L. J., Shoben, E. J., \& Smith, E. E. (1973). Semantic distance and verification of semantic relations. Journal of Verbal Learning \& Verbal Behavior, 12, 1-20.

Rosch, E., \& Mervis, C. B. (1975). Family resemblances: Studies in internal structure of categories. Cognitive Psychology, 7, 573-605.

Rosenberg, S., \& Jones, R. (1972). A method for investigating and representing a person's implicit theory of personality: Theodore Dreiser's view of people. Journal of Personality \& Social Psychology, 22, 372-386.

Rumelhart, D. E., \& Abrahamson, A. A. (1973). Toward a theory of analogical reasoning. Cognitive Psychology, 5, 1-28.

Ruts, W., De Deyne, S., Ameel, E., Vanpaemel, W., Verbeemen, T., \& STORMS, G. (2004). Dutch norm data for 13 semantic categories and 338 exemplars. Behavior Research Methods, Instruments, \& Computers, 36, 506-515. 
Schwanenflugel, P. J., Fabricius, W. V., \& Noyes, C. R. (1996). Developing organization of mental verbs: Evidence for the development of a constructivist theory of mind in middle childhood. Cognitive Development, 11, 265-294.

SchWARZ, G. (1978). Estimating the dimension of a model. Annals of Statistics, 6, 461-464.

SHEPARD, R. N. (1962a). The analysis of proximities: Multidimensional scaling with an unknown distance function. I. Psychometrika, 27, 125-140.

Shepard, R. N. (1962b). The analysis of proximities: Multidimensional scaling with an unknown distance function. II. Psychometrika, 27, 219-246.

SHEPARD, R. N. (1987). Toward a universal law of generalization for psychological science. Science, 237, 1317-1323.

SHEPARD, R. N. (1994). Perceptual-cognitive universals as reflections of the world. Psychonomic Bulletin \& Review, 1, 2-28.

Shoben, E. J., \& Ross, B. H. (1987). Structure and process in cognitive psychology using multidimensional scaling and other techniques. In R. R. Ronning, J. A. Glover, J. C. Conoley, \& J. C. Witt (Eds.), The influence of cognitive psychology on testing. Hillsdale, NJ: Erlbaum.

SMith, J. D., \& Minda, J. P. (1998). Prototypes in the mist: The early epochs of category learning. Journal of Experimental Psychology: Learning, Memory, \& Cognition, 24, 1411-1436.

Smits, T., Storms, G., Rosseel, Y., \& De Boeck, P. (2002). Fruits and vegetables categorized: An application of the generalized context model. Psychonomic Bulletin \& Review, 9, 836-844.

Tversky, A. (1977). Features of similarity. Psychological Review, 84, 327-352.

Verbeemen, T., Vanpaemel, W., Pattyn, S., Storms, G., \& Verguts, T. (2007). Beyond exemplars and prototypes as memory representations of natural concepts: A clustering approach. Journal of Memory \& Language, 56, 537-554.

Verheyen, S., Ameel, E., \& Storms, G. (2007). Determining the dimensionality in spatial representations of semantic concepts. Behavior Research Methods, 39, 427-438.

Wish, M. (1976). Comparisons among multidimensional structures of interpersonal relations. Multivariate Behavioral Research, 11, 297-324.

\section{NOTES}

1. Because this algorithm minimizes distance fits, it was necessary to convert the similarity data into proximities. It has been suggested (e.g., Shepard, 1987, 1994) that similarity is related to psychological distance via an exponential decay function of the form $s_{i j}=\exp \left(-\gamma d_{i j}\right)$; however, it should be noted that arbitrary assumptions about the value of the rate parameter $\gamma$ can have nontrivial effects on the resulting proximities. In light of this, we felt it was safer to convert the similarities into proximities $\left(d_{i j}\right)$ using a simple linear transformation $\left(d_{i j}=1-s_{i j}\right)$.

2. One potential reason for this difference in data precision is the fact that $\bar{\sigma}$ tends to increase as the number of participants increases, and that this increase is most notable when $N$ is small. In order to determine whether the inflated $\bar{\sigma}$ value of the direct-rating data was due to the number of participants included in the calculation (i.e., 15-25 participants completed the direct-rating task for each category, compared with 4 participants per category in the feature applicability task), we calculated $\bar{\sigma}$ on the basis of the average of 10,000 random samples of 4 participants from each category. In this case, the precision was indeed better $(\bar{\sigma}=.14)$, but still above the suggested threshold for a "reasonable" level of interrater variance.

3. Broadly speaking, representational models can be divided into two distinct classes: exemplar models and prototype models. The distancefrom-centroid predictor employed in our analyses falls within the bounds of the prototype class of models. However, following the approach taken in Nosofsky (1988), it is possible to implement an analogous, exemplar model predictor of graded category structure. According to this approach, $\eta_{i}$ in Equation 5 is not measured as the distance between the $i$ th exemplar and the category centroid, but as the average distance between the $i$ th exemplar and all of the other members of the category. Importantly, the results of the analyses on the basis of the exemplar model predictor followed the same pattern of results as the distance-to-centroid model analyses.

4. It should be noted that for the exemplar generation frequency predictions the magnitude of the likelihood differences should be interpreted with some caution. Because of the way that this variable was collected, it had no associated variance. In order to perform the Bayesian analyses, it was necessary to provide an estimate of the data precision, and because this precision assumption has an effect on the overall likelihood, we felt it was safest to assume a uniform, conservative (i.e., relatively imprecise) value of .3 (where the exemplar generation frequency data had been log transformed and $0-1$ normalized). Nonetheless, the ordinal likelihood of the predictions follows the same pattern as the response time predictions, and we can fairly safely assume that the predictions based on the direct-rating representations are at least substantially less likely than those based on the $\mathrm{F}-$ common representations.

(Manuscript received October 14, 2008; revision accepted for publication February 24, 2009.) 